\title{
THE WORLD AS A QUANTUM INFORMATION PROCESSOR
}

\author{
Carlos Eduardo Maldonado \\ Full Professor, School of Medicine \\ Universidad El Bosque, Bogotá, Colombia \\ maldonadocarlos@unbosque.edu.co
}

ORCID: http://orcid.org/0000-0002-9262-8879

\begin{abstract}
It is impossible to fully grasp reality and the universe without a sound understanding of quantum science, i.e. theory. The aim of this paper is twofold, namely first presenting what quantum information processing consists of, and then consequently discussing the implications of quantum science to the understanding of reality. I shall claim that the world is fully quantum, and the classical world is but a limit case of the quantum world. The crux of the argument is that quantum information can be taken as a living phenomenon. Quantum information processing (QIP) has been mainly the subject of computational approaches (Cooper and Hodges, 2016). Here we take it as the way in which information allows for a non-dualistic explanation of the world. In this sense, quantum information processing consists in understanding how entanglement stands as the ground for a coherent reality yet highly dynamical, vibrant and vivid. Information, I argue, is a living phenomenon that creates itself out of nothing. Quantum information is a relational view of entities, systems, phenomena, and events (Auletta, 2005).
\end{abstract}

Keywords: Quantum Information Processing, Living Systems, Non-algorithmic Information, Complexity Theory

\section{Introduction}

This paper argues that the universe or reality can be adequately grasped as a quantum information processor. Two main arguments support the claim, thus: on the one side, quantum information is an interpretation of quantum mechanics (QM), however not equal to any of the other more than fifteen interpretations existing so far; on the other side, the world is fully quantum, and conventional reality is a limiting case of the quantum realm. In order to introduce and discuss these two claims, this paper opens up with a short state-of-the art about the interpretations of QM, and how the door is to be open for quantum information as an interpretation (Auletta, 2005; Fuchs, 2002). 
Accordingly, a discussion with the classical view of reality is necessary to rightly understand what quantum reality is about (Storey, et al., 1995). This is the subject of the second section of this paper in which it is assessed that we live in a quantum world. On this basis, quantum information processing is studied and discussed, and a distance is set out regarding the classical world. The third section argues that the universe or the world is a quantum information processor. We end with a consideration of biological information where it is said that cognition is a biological process; however, previously to the biological processes we have information processing. At the end, some conclusions are drawn.

\section{We live in a quantum world}

Quantum information processing (QIP) has been mainly the subject of computational approaches (Long, 2006). Here we take it as the way in which information allows for a non-dualistic explanation of the world. In this sense, (QIP) consists in understanding how entanglement stands as the ground for a coherent reality yet highly dynamical, vibrant and vivid (Zeilinger, 1999). Information, it will be argued here, is a living phenomenon that creates itself (Vedral, 2010) out of nothing. Quantum information is a relational view of entities, systems, phenomena, and events.

Information is processed in the quantum world as a state of superposition, in terms of non-duality, non-locality, and as entanglement (Li et al., 2016; Vedral, 2003). The processing of information in the quantum realm is an unceasing source of particles, anti-particles, photons, and even phonons in such a way that particles are continuously changed into waves and the waves into particles (Schwindt et al., 1999).

Translated into biological terms, (QIP) can adequately be understood as metabolization i.e. as transforming one thing, say A, into another, say B. Metabolization are processes of energy transformation, storage and release of energy - energy that can be used by other molecules within the same metabolic system. Not ultimately probably the most tenable hypothesis about the origin of life reads: "metabolization first".

Usually when a bond is created bringing forth a larger molecule, energy is stored. Now, when the molecule is broken down energy is released. In any case, energy is never lost, for it can be harnessed by another molecule with the same metabolic network. In a mechanical system the efficiency is low. Yet, when the system is a cell, the efficiency is highly efficient. Such efficiency is due to quantum processes. We can call this as quantum efficiency. In the quantum realm there is no loss of energy, no matter what.

There is an array of interpretations about quantum mechanics (Auletta, 2001). Table No. 1 summarizes them, even though some interpretations are still being developed to-date. 


\section{Table N. 1: Interpretations of Quantum Mechanics}

\begin{tabular}{|l|l|}
\hline \multicolumn{1}{|c|}{ Interpretation } & \multicolumn{1}{|c|}{ Author } \\
\hline Classification adopted by Einstein & Albert Einstein, 1905 \\
\hline $\begin{array}{l}\text { Ensemble interpretation, or statistical } \\
\text { interpretation }\end{array}$ & Max Born, 1926 \\
\hline The Copenhagen interpretation & Niels Bohr, 1927 \\
\hline $\begin{array}{l}\text { von Neumann/Wigner interpretation: } \\
\text { consciousness causes the collapse }\end{array}$ & John von Neumann 1932; Eugene Wigner \\
\hline Quantum logic & $\begin{array}{l}\text { Garrett Birkhoff, John von Neumann, } \\
1936\end{array}$ \\
\hline Broglie-Bohm theory & $\begin{array}{l}\text { Louis de Broglie, 1927; David Bohm, } \\
1952\end{array}$ \\
\hline Many worlds & Hugh Everett, 1957 \\
\hline Stochastic mechanics & Edward Nelson, 1966 \\
\hline Many minds & H. Dieter Zeh, 1970 \\
\hline Modal interpretations of quantum theory & $\begin{array}{l}\text { B. van Fraassen, 1972 and 1974; Bryce } \\
\text { DeWit, 1970;, Dennis Dieks, 1988, and } \\
\text { others }\end{array}$ \\
\hline Consistent histories & Robert Griffiths, 1984 \\
\hline Objective collapse theories & $\begin{array}{l}\text { Girardhi-Rimini-Weber, 1986; Penrose } \\
\text { interpretation, 1989 }\end{array}$ \\
\hline Transactional interpretation & John Cramer, 1986 \\
\hline Relational quantum mechanics & Carlo Rovelli, 1994 \\
\hline Quantum information theories & Charles Bennett, Peter Schor, 1998 \\
\hline Branching space-time theories & Mark Sharlow, 2006 \\
\hline Time-symmetric theories & Ognyan Oreshkov and Niolas Cerf, 2015 \\
\hline The Montevideo Interpretation & Gambini and J. Pullin, 2018 \\
\hline Other interpretations & (9) \\
\hline
\end{tabular}


Source: Own elaboration

From a philosophical standpoint the situation of there being more than seventeen different interpretations about quantum mechanics is very meaningful (Laloë, 2012). Indeed, in the history of science, philosophy and philosophy of science there were usually two rival interpretations; empiricists versus rationalists, for instance. Rather than this being a signal of weakness, it can be taken as a sign of vitality. Something has been going on that suggests a rich variety of interpretations (Fuchs and Peres, 2000). The Holy Grail of science, to so speak, is not the set of observations or data, but the very interpretations of reality, of the models brought out, of the set of data. Interpretation - this is when scientists step on to philosophical issues, as it happens (Halpern, 2018).

Quantum information processing (QIP) can be safely said to be an interpretation of quantum mechanics (Smolin, 2019). It is namely the assessment that the world and the universe are strongly entangled and in a constant superposition (Zwirn, 2017). Moreover, the universe and reality are an unceasing process of transformations (Ellis, 2011) - very much in the tenure of the first law of thermodynamics. As it has been pointed out, quantum coherence breaks down into decoherence, but then decoherence recoheres further on (Maldonado, 2018a). As a consequence, the world is entirely quantum and the conventional or classical reality is just a moment in the dynamics of coherence-decoherence-recoherence. Information, i.e. information processing is the very transformation - say, from plasma to energy to matter to life, in the history of the universe (Walker, et al., 2017).

\section{We live in a quantum world}

From the quantum standpoint, reality is a large realm of processes, fluxes, and dynamics - over against the idea that there are states, objects, and entities. From a philosophical point of view, the classical world has been grasped as a set of equilibria, steady states, and permanence (Modi, et al., 2012). However, from a quantum perspective the universe is a buoyant set of change, becoming, and unceasing creation and destruction of particles and waves (Ball, 2018).

Quantum science has the merit that it introduces a view of the world in a large range, in a deep and wide horizon in which time and space are dissolved, so to speak, and yet can be said to be discrete phenomena. Such a horizon is said to be coherent. Time and space are not distinguished and do not exist as consistent systems. However, quantized time and quantized space remain a puzzle (Rovelli, 2018; Barbour, 1999).

In any case, we do not see the quantum world, for it is highly counter-intuitive. We just grasp it by its effects. The classical world can be said to be the effect of quantum behaviors (Ollivier and Zurek, 2001). Those effects have been traditionally understood as the outcome of the wave collapse. The quantum coherence is broken 
down, it decoheres, and classical reality emerges. This is the standard view of the world.

Nonetheless, there is no wave collapse but a number of instances in which the quantum system decoheres. The classical world is a case limit of the quantum world. Moreover, the classical world is an idealized limiting case of quantum behaviors and phenomena (Li and Luo, 2008).

Due to the advancement of spearhead science at large and the contributions of quantum science in particular, from a cognitive point of view, reality and natural perception do not coincide any longer. Reality is not perceived any more, but imagined, modeled, simulated, or intuited. Along this process reality and the world are conceived not so much in terms of mass (as after the $18^{\text {th }}$ Century) or just as energy (as after the 19 ${ }^{\text {th }}$ Century), but as information (as after the second half of the $20^{\text {th }}$ Century) (cfr. Englert, 1996). Information is a physical stance un-material and intangible, though.

\section{The trouble with the classical view of reality}

The classical world relies entirely on the logical principle of third excluded. This means, the classical world has been taken in terms of either- or - i.e., in terms of a bivalent reality (Mermin, 1985). Dualism is the consequence of such an understanding. Dualism, however, has produced numerous troubles, problems, and unwanted outcomes in the history of science and mankind (Chefles, 2000). Quantum science allows for overcoming a dualist view of the universe.

Indeed, absolutely nothing within the conceptual framework of quantum theory allows for a distinction, and even less an opposition between the microscopic and the macroscopic dimensions (Maldonado, 2019). In the same tenure, ontology and epistemology cease to exist as distinguished stances (Plotnitsky and Khrennikov, 2015). Positively said, epistemology and ontology are one and the same thing in quantum science. Dualism is henceforth untenable.

We cannot simultaneously see or grasp the world as classical and quantum. Suggestive as it is, this means that the standard view of "quantum-like" phenomena is rather a weak argument in that it is flurry or sloppy. We can safely say that classical behavior is a degradation of quantum behaviors. Classical behavior is post-quantum, for at the very beginning of the universe its behavior was quantum. Such idea can be extended to our environmental crisis saying that the degradation of our planet makes it (roughly) classical.

What brings us, indeed, all into peril regarding the climate emergency is not the second law of thermodynamics but the first law because we have not been able to use other forms of energy, and have not been able to change one form of energy into 
another harnessing the various forms of it. The issue that emerges here is quantum thermodynamics.

From the standpoint of cosmology and astrophysics, the classical world emerges when the transition from energy to matter takes place. High energy or temperatures become cooler, and the classical world begins to have a shape, so to speak. Due to the decrease of the initial high temperatures for the new emerging condensate objects temperature becomes a trouble, and it needs to be cooled. The arrow of time arises (Hands, 2017).

The measuring problem was originally considered as the interaction between a quantum and a classical system. This however is not true any longer. To-date a measurement can consist of the interaction among quantum systems, and the measuring apparatus can be a quantum system (Luo, 2008). There are no measures free of interaction (Long, et al., 2014). As a consequence, the measure is always an interaction that entails an entanglement.

There is no need of a conscious agent for a measure to be possible - no matter the nature of the agent. Once we have attained or gained quantum entanglement the measuring problem in the way in which the Copenhagen debate conceived it, disappears: either the wave function is constantly collapsing or else an escape door to the discontinuity of wave function is to be sought. That is exactly what happens with the second quantum revolution, namely quantum information processing, along with several other interpretations of quantum mechanics. The conclusion is that the Copenhagen interpretation is not unique and becomes irrelevant to some extent.

The permanent process that was called the collapse of the wave function becomes now full of jumps or discrete changes; there happen sudden outbreaks unceasingly.

\section{Quantum Information Processing}

Quantum information processing (QIP) can be adequately taken as a living phenomenon on its own. Information is processed by other stances, indeed, but it is also created out of nothing, and consists in an everlasting process of transformation, enrichment, complexification. Information allows for low entropy to remain low and makes that high entropy becomes low making does life possible in the universe. (QIP) can thus be taken as parallel with homeostasis (Damasio, 2018), which is not quite different to metabolization.

The world has not been created by any creator, but it can be said to be its own work, a non-linear, surprising and emerging world, though. Indeed, taking up the world as a quantum information processor firmly sets the ground for self-organization and selfcatalytic sets or loops.

A number of consequences follow from the considerations above. Probably the most puzzling one is the fact that the doors to panpsychism are being wide open 
(Maldonado, 2018b). Pansychism, namely, the assumption that consciousness pervades reality from one extreme to the other, can also be understood as a sort of pantheism; in other words, the idea that life is not just a particular case in the universe. On the contrary, ultimately, the universe itself is alive.

The idea can be expressed in computational terms saying that the universe does compute (Lloyd, 2006). Or also, that the information is processed from the abiotic stance on to the biotic one, even though in a variety of ways. Panpsychism, pantheism, biocentrism, or also hylozoism, have never encountered a starring role in the history of science or philosophy. A most compelling consequence, as it happens.

The world is an information processor radically quantum. Quantum behaviors ground the very shape, expressions and behaviors of the world at large.

Information - and not any longer just matter or energy - can be viewed as the physical concept that best captures the nature of the world, namely processing, change, and creation. Yet, information does not exist before the processing, and neither after processing. Information exists as and in the moment of the processing.

Living beings and the universe do not just read the environment, if allowed. Besides and even better, they continuously create new information. Information is created in a manifold of ways, thus: as behaviors, signs, networks, actions or more information. We are then faced with an increasing complexity, which is the landmark of the world in general. We all live in an increasingly complex universe that creates and transforms information unceasingly. The universe is anew at each moment, once and again (Kauffman, 2016).

One more puzzling consequence is the recognition that new information corresponds to new surprises. Indeed, the meaning of information is proportional to the randomness and surprise that are entailed. The lower the randomness and surprise, the more predictable information is (Vedral, 2010). Unpredictable information points out straightforwardly to novelty, ingeniosity, and creativity. The universe is the name for the creation and appearance of new information - quantum information processing.

Hereafter, the non-deterministic behavior of the world manifests itself in many aspects.

\section{Biological information}

Translated into biological terms, (QIP) can adequately be understood as metabolization i.e. as transforming one thing, say A, into another, say B. Metabolization are processes of energy transformation, storage and release of energy - energy that can be used by other molecules within the same metabolic system (Djordjevic, 2016). Usually when a bond is created bringing forth a larger molecule, 
energy is stored. Now, when the molecule is broken down energy is released. In any case, energy is never lost, for it can be harnessed by another molecule with the same metabolic network. In a mechanical system the efficiency is low. Yet, when the system is a cell, the efficiency is highly efficient. Such efficiency is due to quantum processes. We can call this as quantum efficiency.

In the quantum realm there is no loss of energy, no matter what. Now, the fact that there is no loss of energy can be more adequately stated saying that there is no loss of information. Information transforms itself into other forms of information, and thus, metabolization and life are unceasing ever-lasting processes and dynamics; i.e. complex dynamics.

It can be safely said that cognition is a biological process, and yet, previously to the biological processes we have information processing (Auletta, 2011). Information does not exist before it is processed, and does not exist after it is processed. Information is the very outcome of its being processed. Now, the processing means its change and transformation, which go on from the very stage in which an atom is excited and goes inwards or outwards of its orbit, up to the very enriching, transformation, and change in and by living beings (Maldonado, Gómez-Cruz, 2015b).

Given the distinction between classical information and quantum information, it is more than reasonable to assess that information is quantum and that it has been studied thoroughly in a number of biological levels and processes (birds migration, magnetic field of the Earth, the human brain, and many other cases). Quantum effects are now widely recognized at the macroscopic level in living beings (Hobson, 2013).

\section{Conclusions}

There are not hyletic (or material) differences among biotic and abiotic stances (Walker et al., 2017). The differences can be said to be only qualitative, of degrees or of organization. Reality as a whole is a process of unceasing unfolding and reconfiguration. The main title of such an unfolding can be seen as coherencedecoherence-recoherence. Somewhere in the midst of such a process the classical or conventional world emerges - as an idealized limit of the quantum world.

In the hardcore of quantum science there is not one single argument allowing juxtapose the microscopic and the macroscopic dimensions of the world. They are just a difference of time-speed, thus: the macroscopic world is slow whereas the microscopic one is rapid and increasingly vertiginous.

Ultimately the very origin of life consists in processing information, i.e. processing quantum information. The issue that emerges here apparently points to the mathematics of symmetry. This point sets out the horizon for further research and is to be left here aside.

\section{References}


Auletta, A., (2001). Foundations and interpretation of Quantum Mechanics. World Scientific Publishing

Auletta, G., (2005). "Quantum information as a general paradigm”, in: Found. Phys. 35, 787

Auletta, G., (2011). Cognitive biology. Cambridge: Cambridge University Press

Ball, Ph., (2018). Beyond Weird. Why everything you thought yu knew about quantum physics is different. The Bodley Head

Barbour, J., (1999). The End of Time. The Next Revolution in Physics. Oxford: Oxford University Press

Chefles, A., (2000). “Quantum state discrimination”, in: Contemp. Phys. 41, 401

Damasio, A., (2019). The Strange Order of Things: Life, Feeling, and the Making of Cultures. Penguin Books

Djordjevic, I. B., (2016). Quantum Biological Information Theory. Springer Verlag

Cooper, S. B., and Hodges, A., (2016). The Once and Future Turing. Cambridge: Cambridge University Press

Ellis, G. F. R., (2011). “Does the multiverse really exist?”, in: Scientific American, August issue, pp. 38-43

Englert, B. G., (1996). "Fringe Visibility and Which-Way Information: An Inequality", in: Phys. Rev. Lett. 77, 2154

Fuchs, C. A., and Peres, A., (2000). "Quantum theory needs no 'interpretation", in: Physics Today 53, March 2000, 70-71, doi: https://doi.org/10.1063/1.883004

Fuchs, Ch., (2002). "Quantum Mechanics as Quantum Information (and a little bit more)", in: https://arxiv.org/pdf/quant-ph/0205039.pdf

Halpern, P., (2018). The Quantum Labyrinth. How Richard Feynman and John Wheller Revolutionized Time and Reality. New York: Basic Books

Hands, J., (2017). Cosmosapiens. Human Evolution from the Origin of the Universe. New York-London: Overlook Duckworth

Hobson, A., (2013). “There are no particles, only fields”, in: Am. J. Phys. 81, 211

Kauffman, S., (2016). Humanity in a Creative Universe. Oxford: Oxford University Press

Laloë, F., (2012). Do we really understand quantum mechanics. Cambridge: Cambridge University Press 
Li, N., and Luo, S. L., (2008). "Classical states versus separable states", in: Phys. Rev. A 78, 024303

Luo, S. L., (2008). "Using measurement-induced disturbance to characterize correlations as classical or quantum", in: Phys. Rev. A 77, 022301

Lloyd, S., (2006). Programming the Universe. A Quantum Computer Scientist Takes on the Cosmos. New York: Alfred A. Knopf

Long, G. L., (2006). “General quantum interference principle and duality computer”, in: Communications in Theoretical Physics 45, 825-844

Long, G. L., Qin, W., Yang, Z., and Li, J. L., (2014). "Realistic Interpretation of Quantum Mechanics and Encounter-Delayed-Choice Experiment”, in: arXiv: 1410.4129

Maldonado, C. E., (2019) "Quantum Theory and the Social Sciences", in: Momento. Revista de Física, 59E, Oct., pp.. 34-47; https://doi.org/10.15446/mo.n59E.81645

Maldonado, C. E., (2018a). "A Quantum Coherence-Recoherence-Based Model of Reality", en: Neuroquantology, 16(11): 44-48, doi: 10.14704/nq.2018.16.11.1858; available at: https://www.neuroquantology.com/index.php/journal/article/view/1858/1285

Maldonado, C. E., (2018b) "Quantum Physics and Consciousness: A (Strong) Defense of Panpsychism", en: Trans/from/acao, Edicao especial, Vol. 41, pp. 101-118, doi: http://dx.doi.org/10.1590/0101-3173.2018.v4lesp.07.p101

Maldonado, C. E., Gómez-Cruz, N., (2015) "Biological Hypercomputation: A New Research Problem in Complexity Theory”, en: Complexity, Vol. 20, Issue 4, págs. 8-18

Mermin, N. D., (1985). "Is the moon there when nobody looks? Reality and the quantum theory", in: Phys. Today 38 (4), 38

Modi, K., Brodutch, A., Cable, H., Paterek, T., and Vedral, V., (2012). "The classicalquantum boundary for correlations: Discord and related measures", in: Rev. Mod. Phys. 84, 1655

Ollivier, H., and Zurek, W. H., (2001). "Quantum Discord: A Measure of the Quantumness of Correlations", in: Phys. Rev. Lett. 88, 017901

Plotnitsky, A., and Khrennikov, A., (2015). "Reality without Realism: On the Ontological and Epistemological Architecture of Quantum Mechanics", in: Found. Phys. 45, 1269

Rovelli, C., (2018). The Order of Time. Riverhead Books

Ruyant, K., (2018). “Can we make sense of relational quantum mechanics?”, in: Found. Phys. 48, 440 
Schwindt, P. D. D., Kwiat, P. G., and Englert, B. G., (1999). “Quantitative wave-particle duality and nonerasing quantum erasure", in: Phys. Rev. A 60, 4285

Smolin, L., (2019). Einstein's Unfinished Revolution. The Search for What Lies Beyond the Quantum. New York: Penguin Press

Storey, E. P., Tan, S. M., Collett, M. J., and Walls, D. F., (1995). “Complementarity and uncertainty", in: Nature (London) 375, 368

Vedral, V., (2010). Decoding Reality. The Universe as Quantum Information. Oxford: Oxford University Press

Vedral, V., (2003). "Classical Correlations and Entanglement in Quantum Measurements", in: Phys. Rev. Lett. 90, 050401

Zeilinger, A., (1999). "A foundational principle for quantum mechanics”, in: Found. Phys. 29, 639

Zwirn, H., (2017). “Delayed Choice, Complementarity, Entanglement and Measurement", in: Phys. Essays 30, 281

Walker, S. I., Davies, P. C. W., Ellis, G. F. R. (Eds)., (2017). From Matter to Life. Information and Causality. Cambridge: Cambridge University Press 